\title{
Cutaneous findings in children and adolescents with attention deficit and hyperactivity disorder
}

\author{
Nihal Altunisik ${ }^{1}$, Ilknur Ucuz ${ }^{1}$, Dursun Turkmen ${ }^{1}$, and Serpil Sener ${ }^{1}$ \\ ${ }^{1}$ Inonu Universitesi Tip fakultesi
}

April 28, 2020

\begin{abstract}
Background/Aim: Attention Deficit and Hyperactivity Disorder (ADHD) is the most frequently diagnosed psychiatric disorder in children. Only one study was found in literature which examined cutaneous findings in ADHD so far. The aim of the present study is to evaluate the cutaneous findings of children and adolescents diagnosed with ADHD and to compare the incidence of these findings with healthy controls. Methods: Forty-six patients diagnosed with ADHD according to The Diagnostic and Statistical Manual of Mental Disorders fifth edition (DSM-V) criteria and 50 healthy controls with no ADHD symptoms who had similar characteristics to the patient group in terms of sociodemographic characteristics such as age and gender were included in the study. All the patients underwent whole body dermatological examination and pathological findings in the skin, hairy skin and nails in the patient and control group were recorded. Results: The most common finding in the patient group was found as onychophagy and/or periungual skin biting with a rate of $76.1 \%$. In addition, statistically significant difference was found between the two groups in terms of xerosis, prurigo and allergy history. Conclusions: It is important to be careful in terms of cutaneous findings that may accompany ADHD in terms of both early treatment and also for preventing possible complications. In addition, determination of these findings will guide future studies in terms of identifying the common aetiology of ADHD and some cutaneous diseases.
\end{abstract}

\section{What's already known about this topic?}

-ADHD is one of the most frequent developmental disorders of the childhood.

-Studies and case reports have reported that dermatological diseases such as atopic dermatitis and acne and cutaneous findings called skin picking symptoms are more common in children and adolescents diagnosed with ADHD.

\section{What does this article add?}

-The most common finding in the patient group was found as onychophagy and/or periungual skin biting with a rate of $76.1 \%$.

-In addition, statistically significant difference was found between the two groups in terms of xerosis, prurigo and allergy history.

\section{Introduction}

Attention Deficit and Hyperactivity Disorder (ADHD) is the most frequently diagnosed psychiatric disorder in children and it is characterized with shortness of persistent and continuous attention span, agility and restlessness in behaviours or cognition due to lack of control to prevent. These children have carelessness or excessive activity that does not match the developmental period they are in. In studies conducted in our country, its prevalence in population samples was found to differ between $8.6 \%$ and $8.1 \%$, while its prevalence 
in clinical samples was found to differ between $8.6 \%$ and $29.44 \%$ (1). While organic and social reasons are emphasized in the occurrence of the disorder, its aetiology is not completely known (2).

ADHD is one of the most frequent developmental disorders of the childhood. ADHD, which brings additional costs to the society apart from treatment and the effects of which last for a lifetime, has become an increasing focus of attention in recent years. Although a high comorbidity has been reported with other psychiatric disorders, it has also been found that ADHD may coexist with allergic and neurological diseases, obesity and eating disorders, traumatic injuries, risky sexual behaviours and sleep disorders (3). Studies and case reports have reported that dermatological diseases such as atopic dermatitis and acne and cutaneous findings called skin picking symptoms are more common in children and adolescents diagnosed with ADHD (4-6). However, only one study has been found in literature investigating cutaneous findings in ADHD (7). The aim of the present study is to assess cutaneous findings in children and adolescents diagnosed with ADHD and to compare the incidence of these findings with healthy controls.

\section{Materials and Methods}

Forty-six patients diagnosed with ADHD in paediatric and adolescent psychiatry clinic according to The Diagnostic and Statistical Manual of Mental Disorders fifth edition (DSM-V) criteria and 50 healthy controls with no ADHD symptoms who had similar characteristics to the patient group in terms of sociodemographic characteristics such as age and gender were included in the study. In the power analysis, when $\alpha: 0.05,1-\beta$ (power): 0.80 was taken, it was calculated that at least 42 subjects should be taken from each group in order to have a $19.7 \%$ difference in the number of nail-eating (onychophagia) compared to the control group in the patient group with attention deficit hyperactivity disorder. The patient and the control group were evaluated by a dermatologist in terms of the detection of comorbid cutaneous findings and dermatological diseases. All the patients underwent whole body dermatological examination and pathological findings in the skin, hairy skin and nails in the patient and control group were recorded.

The study was conducted in accordance with the Helsinki Declaration. Local ethical board approval was taken for the study. SPSS (Statistical Package for Social Sciences) for Windows 22.0 program was used for the statistical analysis of the data obtained from the study. The values obtained from the study were presented as average \pm standard deviation or number and percentage. Chi-square test was used in statistical assessments. Level of significance was taken as $\mathrm{p}<0,05$ for all results.

\section{Results}

A total of 46 patients, 35 males and 11 females, diagnosed with ADHD were included in the study, while a total of 50 individuals, 33 males and 17 females, were included as the control group. Average age of the patient group was $9.6 \pm 1.9$ (min-max: 6-15), while the average age of the control group was $10.4 \pm 2.9$ (minmax: 4-16). There were no statistically significant differences between the patient and the control group in terms of age and gender $\left(\mathrm{p}_{i}, 0.05\right)$.

Table 1 shows the cutaneous findings of the ADHD patients and control group. The most frequent finding in the patient group is onychophagy and/or periungual skin biting with a rate of $76.1 \%$. This rate was found as $14 \%$ in the control group and the difference between the two groups was found to be statistically significant. Statistically significant difference was also found in terms of xerosis, prurigo and allergy between the two groups. Acne was found to be statistically significantly higher in the control group when compared with the patient group. No statistically significant difference was found between the two groups in terms of other findings.

\section{Discussion}

Only one study evaluating cutaneous findings was found in literature in patients diagnosed with ADHD. No studies were found which were conducted comparatively with a control group. In a study by Erdoğan et al. (7), 68 children and adolescents diagnosed with ADHD were evaluated in terms of cutaneous findings. In dermatological examination, at least one cutaneous finding was determined in $88.2 \%$ of the cases. They reported that the most frequent finding was nail biting (onychophagy). Xerosis was found in 22 (32.3\%) 
cases, acne was found in $11(16.2 \%)$ cases, chronic traumatic dermatological changes were found in $12(17.6 \%)$ cases and atopic dermatitis was found in $7(10.3 \%)$ cases.

In our study, the most frequent finding in our patient group was onychophagy and/or removal of the skin around nail. Onychophagy, which is defined as habitual nail biting, is a common disease which influences 20$30 \%$ of the population and all age groups. This situation can cause psychosocial problems and complications that include both nail unit and the mouth cavity (8). In one study conducted, it was reported that the most frequent comorbid disease in children and adolescents who bit their nails was ADHD with a rate of $74.6 \%$ (9). It was thought that the increase in predisposition to impulsive behaviour caused the detection of nail biting behaviour in high rates. In addition, the result that higher rates of multiple scars were found in the patient group, although not in statistically significant levels, supports that the patients are prone to impulsive behaviour and that hyperactivity increases exposure to trauma.

Skin picking disorder (SPD) includes behaviours such as impulsive, repetitive picking, scratching and tightening of the normal skin although there is no dermatological condition. Skin picking is also called dermatillomania, skin excoriation or psychogenic excoriation. This situation causes important disorders in patients' daily lives and psychosocial functions. In addition, important medical complications can occur such as infections, scarring and serious physical deformity (6). Cases with coexistence of SPD and ADHD were reported in literature. In these cases, improvement was found in SPD symptoms with methylphenidate used in ADHD treatment $(6,10)$. Another view is that since behaviours such as skin picking, trichotillomania, nail biting and chewing lips or cheek mucosa are similar to each other phenomonologically, they should be grouped as "body oriented repetitive behaviour" (11). SPD was found in two cases diagnosed with ADHD in our study. However, trichotillomania, which is another impulsive psychiatric disorder, was not found in any cases or in individuals in the control group.

In our study, other dermatological pathologies found in patients with ADHD were xerosis, prurigo and atopic dermatitis (AD). There are a few hypotheses explaining the comorbidity between AD and ADHD. The first one is that the neuroimmune pathways supported with some experimental evidence in both diseases may be responsible for this relationship. It is thought that in early childhood, when the brain is not fully mature, the effects of allergic inflammatory cytokines can be more obvious. The second hypothesis is that ADHD may be a behavioural response to itching or discomfort resulting from comorbid psychological processes. The third hypothesis is that ADHD is closely correlated with other mental disorders which have comorbidity with AD (12). Genuneit et al. (4) found a strong correlation between ADHD and AD in early childhood. Hak et al. (13) reported that in childhood ADHD is correlated with atopic diseases and impetigo. While AD was found in only two cases in our study, xerosis, prurigo was found to be statistically significantly higher in cases with ADHD when compared with the control group.

Environmental risk factors that provoke allergic reactions such as some food allergens play a role in the increase of both ADHD and allergic asthma symptoms. This findings show that in addition to a strong genetic predisposition, increasingly more exposure to environmental factors can contribute to the development of ADHD and allergic asthma. However, possible etiologic ways are not fully understood yet (13). When the cases were examined in terms of allergy history in our study, statistically significantly higher positivity was found in patients diagnosed with ADHD. Presence of xerosis, pruritus and allergy history are findings suggestive of atopy. This result brought to mind that patients have atopic cutaneous findings in ADHD even if atopic dermatitis lesions are not found.

In a study they conducted, Gupta et al. (5) researched the prevalence of ADHD in acne patients and found a strong correlation between acne and ADHD. They emphasized that ADHD could contribute to psychiatric morbidity and suicidal intention in acne patients. Unlike this study, we found statistically significantly higher acne in control group.

As a conclusion, we determined the highest observed cutaneous findings in ADHD, which is a common psychiatric disease in children. The limitation of our study is the fact that the number of patients and controls was relatively limited. Including a control group can be accepted as the positive aspect of our study. 
However, studies with higher number of cases and multiple centres are required to reach more objective data since there are few studies conducted on the issue. This way, care will be taken in terms of skin diseases that may accompany this disease group. With early treatment methods, cutaneous complications that may occur in the future will also be prevented. In addition, the data will contribute to literature in terms of finding out the common aetiology of ADHD and some cutaneous diseases.

\section{References}

1. Erșan EE, Doğan O, Doğan S, Sümer H. The distribution of symptoms of Attention Deficit/ Hyperactivity Disorder and Oppositional Defiant Disorder in school age children in Turkey. Eur Child Adolesc Psychiatry 2004; 13: 354-61.

2. Öncü B, Şenol S. The Etiology of Attention Deficit Hyperactivity Disorder: An Integrative Approach Klinik psikiyatri 2002; 5: 111-9.

3. Tufan AE, Yaluğ İ. Medical Comorbidity in Attention Deficit Hyperactivity Disorder. Current Approaches in Psychiatry 2009; 1: 187-200.

4. Genuneit J, Braig S, Brandt S, et al. Infant atopic eczema and subsequent attention-deficit/hyperactivity disorder-a prospective birth cohort study. Pediatr Allergy Immunol 2014; 25: 51-56.

5. Gupta MA, Gupta AK, Vujcic B. Increased frequency of Attention Deficit Hyperactivity Disorder (ADHD) in acne versus dermatologic controls: analysis of an epidemiologic database from the US $J$ Dermatolog Treat . 2014; 25: 115-8.

6. Çolak Sivri R, Çolak B. Cessation of Skin Picking Symptoms With Methylphenidate Treatment in a Child With Comorbid Skin Picking and Attention-Deficit/Hyperactivity Disorder. Clin Neuropharmacol2019; 42: 105-7.

7. Erdogan HK, Fidan ST, Bulur I, Karapınar T, Saracoglu ZN. Evaluation of Cutaneous Findings in Children and Adolescents with Attention Deficit Hyperactivity Disorder: A Preliminary Study. Pediatr Dermatol2017; 34: 93-4.

8. Halteh P, Scher RK, Lipner SR. Onychophagia: A nail-biting conundrum for physicians. Dermatolog Treat 2017; 28: 166-72.

9. Ghanizadeh A. Association of nail biting and psychiatric disorders in children and their parents in a psychiatrically referred sample of children. Child Adolesc Psychiatry Ment Health $2008 ; 2$ : 13.

10. Bernardes C, Mattos P, Nazar BP. Skin picking disorder comorbid with ADHD successfully treated with methylphenidat. Braz J Psychiatry2018; 40: 110-1.

11. Bohne A, Wilhelm S, Keuthen NJ, Baer L, Jenike MA. Skin picking in German students: prevalence, phenomenology and associated characteristics. Behav Modif 2002; 26: 320-39.

12. Buske-Kirschbaum A, Schmitt J, Plessow F, Romanos M, Weidinger S, Roessner V. Psychoendocrine and psychoneuroimmunological mechanisms in the comorbidity of atopic eczema and attention deficit/hyperactivity disorder. Psychoneuroendocrinology 2013: 38: 12-23.

13. Hak E, de Vries TW, Hoekstra PJ, Jick SS. Association of childhood attention-deficit/hyperactivity disorder with atopic diseases and skin infections? A matched case-control study using the General Practice Research Database. Ann Allergy Asthma Immunol 2013; 111: 102-6.

Table 1. Cutaneous Findings in the patient and control groups 


\begin{tabular}{|c|c|c|c|c|}
\hline Finding & Finding & Patient group n (\%) & $\begin{array}{l}\text { Control group N } \\
(\%)\end{array}$ & $\mathrm{p}$ \\
\hline $\begin{array}{l}\text { Onychophagy } \\
\text { and/or periungual } \\
\text { skin biting }\end{array}$ & Yes No & $35(76.1) 11(23.9)$ & $7(14) 43(86)$ & $<0.001$ \\
\hline Xerosis & Yes No & $20(43.5) 26(56.5)$ & $10(20) 40(80)$ & 0.024 \\
\hline Prurigo & Yes No & $13(28.3) 33(71.7)$ & $1(2) 49(98)$ & 0.001 \\
\hline Leukonychia & Yes No & $9(19.6) 37(80.4)$ & $6(12) 44(88)$ & 0.460 \\
\hline Allergy history & Yes No & $8(17.4) 38(82.6)$ & $2(4) 48(96)$ & 0.045 \\
\hline Multiple scars & Yes No & $6(13) 40(87)$ & $1(2) 49(98)$ & 0.052 \\
\hline Acne & Yes No & $2(4.3) 44(95.7)$ & $11(22) 39(78)$ & 0.026 \\
\hline Verruca & Yes No & $0(0) 46(100)$ & $2(4) 48(96)$ & 0.496 \\
\hline Urticaria & Yes No & $3(6.5) 43()$ & $0(0) 50(100)$ & 0.106 \\
\hline Hemangioma & Yes No & $2(4.3) 44(95.7)$ & $1(2) 49(98)$ & 0.606 \\
\hline $\begin{array}{l}\text { Hyperhidrosis of the } \\
\text { hand and/or foot }\end{array}$ & Yes No & $2(4.3) 44(95.7)$ & $4(8) 46(92)$ & 0.679 \\
\hline Atopic dermatitis & Yes No & $2(4.3) 44(95.7)$ & $0(0) 50(100)$ & 0.227 \\
\hline $\begin{array}{l}\text { Skin picking } \\
\text { disorder }\end{array}$ & Yes No & $2(4.3) 44(95.7)$ & $0(0) 50(100)$ & 0.227 \\
\hline Lip lick dermatitis & Yes No & $1(2.2) 45(97.8)$ & $1(2) 49(98)$ & 1.00 \\
\hline Pityriasis versicolor & Yes No & $1(2.2) 45$ & $0(0) 50(100)$ & 0.479 \\
\hline $\begin{array}{l}\text { Congenital } \\
\text { hypopigmented } \\
\text { patch }\end{array}$ & Yes No & $1(2.2) 45(97.8)$ & $0(0) 50(100)$ & 0.479 \\
\hline Ephalides in face & Yes No & $1(2.2) 45(97.8)$ & $2(4) 48(96)$ & 1.00 \\
\hline $\begin{array}{l}\text { Seborrheic } \\
\text { dermatitis }\end{array}$ & Yes No & $2(4.3) 44(95.7)$ & $4(8) 46(92)$ & 0.679 \\
\hline Nail pitting & Yes No & $1(2.2) 45(97.8)$ & $0(0) 50(100)$ & 0.479 \\
\hline Pityriasis alba & Yes No & $1(2.2) 45(97.8)$ & $0(0) 50(100)$ & 0.479 \\
\hline $\begin{array}{l}\text { Congenital } \\
\text { hyperpigmented } \\
\text { patch }\end{array}$ & Yes No & $1(2.2) 45(97.8)$ & $0(0) 50(100)$ & 0.479 \\
\hline
\end{tabular}

